Short Note

\title{
Revisit to the THINC scheme: A simple algebraic VOF algorithm
}

\author{
Feng Xiao ${ }^{\mathrm{a}, \mathrm{b}, *}$, Satoshi $\mathrm{Ii}^{\mathrm{c}}$, Chungang Chen ${ }^{\mathrm{b}}$ \\ ${ }^{a}$ Department of Energy Sciences, Tokyo Institute of Technology, 4259 Nagatsuta, Midori-ku, Yokohama 226-8502, Japan \\ ${ }^{\mathrm{b}}$ LHD, Institute of Mechanics, CAS, No. 15 Beisihuanxi Road, Haidian District, Beijing 100190, China \\ ${ }^{\mathrm{c}}$ Department of Mechanical Engineering, The University of Tokyo, 7-3-1 Hongo Bunkyo-ku, Tokyo 113-8656, Japan
}

\section{A R T I C L E I N F O}

\section{Article history:}

Received 3 February 2011

Received in revised form 3 May 2011

Accepted 10 June 2011

Available online 23 June 2011

\section{Keywords:}

Free interface

Interface capturing/tracking

VOF

Hyperbolic tangent function

Multi-dimensional calculation

Multi-phase flows

\begin{abstract}
A B S T R A C T
This short note presents an improved multi-dimensional algebraic VOF method to capture moving interfaces. The interface jump in the THINC (tangent of hyperbola for INterface capturing) scheme is adaptively scaled to a proper thickness according to the interface orientation. The numerical accuracy in computing multi-dimensional moving interfaces is significantly improved. Without any geometrical reconstruction, the proposed method is extremely simple and easy to use, and its numerical accuracy is superior to other existing methods of its kind and comparable to the conventional PLIC (piecewise linear interface calculation) type VOF schemes.
\end{abstract}

(c) 2011 Elsevier Inc. All rights reserved.

\section{The THINC scheme}

VOF (volume of fluid) method has been widely used to compute moving interfaces. The essential part of a conventional VOF method is the cell-wise geometrical reconstruction of the interface that divides the cell into domains of different fluids. The geometrical information of the reconstruction is then used to compute the numerical fluxes for solving the following transport equation of the volume fraction $\phi$ of a certain fluid,

$$
\frac{\partial \phi}{\partial t}+\nabla \cdot(\mathbf{u} \phi)-\phi \nabla \cdot \mathbf{u}=0
$$

where $\mathbf{u}$ is the velocity field, and the VOF function $\phi$ has a value between 0 and 1 .

The algorithm for geometrically reconstructing the interface affects the quality of the numerical solutions, and it remains so far an active topic. Recent progresses of the VOF schemes are achieved along the path of the PLIC (piecewise linear interface calculation) reconstruction $[1-7,12,13,15,18]$. However, a geometrical reconstruction fatalistically makes the computer coding complicated and filled with "if" logic operations, which usually prevents them from being immediately adoptable to the users. A VOF algorithm that does not need any geometrical reconstruction, referred to as algebraic type VOF method in our context, should be of practical interest. Following the existing works [14,11], we devised a VOF scheme, so called THINC (tangent of hyperbola for INterface capturing) [16], for computing moving interfaces without the geometrical reconstruction. The original THINC scheme effectively maintains the interface thickness but tends to ruffle the surface when the flow direction is almost parallel to the interface. A significant improvement in the interface shape faithfulness can be made by blending the THINC scheme and the first-order unwind advection scheme [19].

\footnotetext{
* Corresponding author at: Department of Energy Sciences, Tokyo Institute of Technology, 4259 Nagatsuta, Midori-ku, Yokohama 226-8502, Japan.

E-mail address: xiao@es.titech.ac.jp (F. Xiao).
} 
In this short note, we further explore the improved multi-dimensional VOF calculation by making use of the attractive feature of the 1D THINC building block. The resulting scheme, so called THINC/SW (THINC with Slope Weighting), is extremely simple, yet its numerical accuracy is comparable to the conventional VOF schemes that use geometrical reconstructions.

The basic 1D THINC scheme is devised for the following advection equation of the VOF function

$$
\frac{\partial \phi}{\partial t}+\frac{\partial}{\partial x}(u \phi)-\phi \frac{\partial u}{\partial x}=0
$$

Given the cell-averaged values of the numerical solution to (2) defined piece-wisely for mesh cell $\left[x_{i-\frac{1}{2}}, x_{i+\frac{1}{2}}\right]$ at the $n$th time step $\left(t=t^{n}\right)$,

$$
\bar{\phi}_{i}^{n}=\frac{1}{\Delta x_{i}} \int_{x_{i-\frac{1}{2}}}^{x_{i+\frac{1}{2}}} \phi\left(x, t^{n}\right) d x
$$

with $\Delta x_{i}=x_{i+\frac{1}{2}}-x_{i-\frac{1}{2}}$, we compute the solution at the $(n+1)$ th time step by the finite volume formulation,

$$
\bar{\phi}_{i}^{n+1}=\bar{\phi}_{i}^{n}-\left(f_{i+\frac{1}{2}}-f_{i-\frac{1}{2}}\right) / \Delta x_{i}+\bar{\phi}_{i}^{n}\left(u_{i+\frac{1}{2}}-u_{i-\frac{1}{2}}\right) \Delta t / \Delta x_{i}
$$

where $f_{i+\frac{1}{2}}$ is the flux transported across boundary $x=x_{i+\frac{1}{2}}$ during $\Delta t=t^{n+1}-t^{n}$, and computed by

$$
f_{i+\frac{1}{2}}= \begin{cases}-\int_{t^{n}}^{t^{n+1}} \Phi_{i}\left(x_{i+\frac{1}{2}}-u_{i+\frac{1}{2}}\left(t-t^{n}\right)\right) u d t, & \text { if } u_{i+\frac{1}{2}} \geqslant 0 \\ \int_{t^{n}}^{t^{n+1}} \Phi_{i+1}\left(x_{i+\frac{1}{2}}-u_{i+\frac{1}{2}}\left(t-t^{n}\right)\right) u d t, & \text { otherwise }\end{cases}
$$

where $\Phi_{i}(x)$ is the reconstruction interpolation function. The THINC scheme uses the piecewise hyperbolic tangent function

$$
\Phi_{i}(x)=\frac{1}{2}\left(1+\gamma \tanh \left(\beta\left(\frac{x-x_{i-\frac{1}{2}}}{\Delta x_{i}}-\tilde{x}_{i}\right)\right)\right),
$$

where $\gamma=1$ for $\phi_{i-1}<\phi_{i+1}$ and $\gamma=-1$ for $\phi_{i-1}>\phi_{i+1}$. $\beta$ is a prescribed parameter to control the slope and the thickness of the jump, and will be discussed in the next section. The only unknown in (6) is the jump center $\tilde{x}_{i}$ which is uniquely determined for the given $\bar{\phi}_{i}^{n}$ by solving $\frac{1}{\Delta x_{i}} \int_{x_{i-1}}^{x_{i} \frac{1}{2}} \Phi_{i}(x) d x=\bar{\phi}_{i}^{n}$.

For user's convenience, we give explicitly some formula to calculate the numerical flux as below:

- Set $\lambda=0$ and iup $=i+1$ for $u_{i+\frac{1}{2}}<0$, or $\lambda=1$ and iup $=i$ otherwise;

- Calculate the jump location of the upwind cell $\tilde{x}_{\text {iup }}$ by

$$
\tilde{x}_{\text {iup }}=\frac{1}{2 \beta} \ln \left(\frac{e^{\left.\frac{[\beta}{\gamma}\left(1+\gamma-2 \bar{\phi}_{\text {iup }}\right)\right]}-1}{1-e^{\left[\frac{\beta}{\gamma}\left(1-\gamma-2 \bar{\phi}_{i u p}\right)\right]}}\right)
$$

- Calculate the numerical flux by

$$
f_{i+\frac{1}{2}}=\frac{1}{2}\left(-u_{i+\frac{1}{2}} \Delta t+\frac{\gamma \Delta x}{\beta} \ln \left(\frac{\cosh \left[\beta\left(\lambda-\tilde{x}_{\text {iup }}-u_{i+\frac{1}{2}} \Delta t / \Delta x\right)\right]}{\cosh \left[\beta\left(\lambda-\tilde{x}_{\text {iup }}\right)\right]}\right)\right) .
$$

The multi-dimensional computation can be conducted by the operator splitting using the $1 \mathrm{D}$ scheme as the building block.

\section{The thickness of jump transition}

We have run a number of numerical experiments to transport a 1D step-like jump with different $\beta$ for long time computation ( $10^{4}$ time steps), and obtained very interesting observations as follows. (i) THINC scheme is essentially different from other Eulerian high resolution advection schemes and is completely free from numerical diffusion that smears out steepness in the solutions, (ii) The thickness of a jump in the numerical solution maintains constant even for long time computation and is independent of the CFL number, and (iii) The interface jump can be scaled to a predetermined thickness by controlling the value of $\beta$.

Fig. 1 (left), for example, gives the computed jumps after $10^{4}$ time steps with $\beta=0.2$ and 2.3 respectively. It is shown that the computed jump thickness can be effectively controlled by $\beta$.

In order to quantitatively estimate the thickness of the transition zone of the jump represented by (6), we define the jump transition zone as the region where $\epsilon \leqslant \Phi_{i}(x) \leqslant 1-\epsilon$ holds with $\epsilon$ being a small positive number $(\epsilon=0.001)$. We examined the thickness of the jump transition zone computed by the THINC scheme for different $\beta$ values and counted the number of 

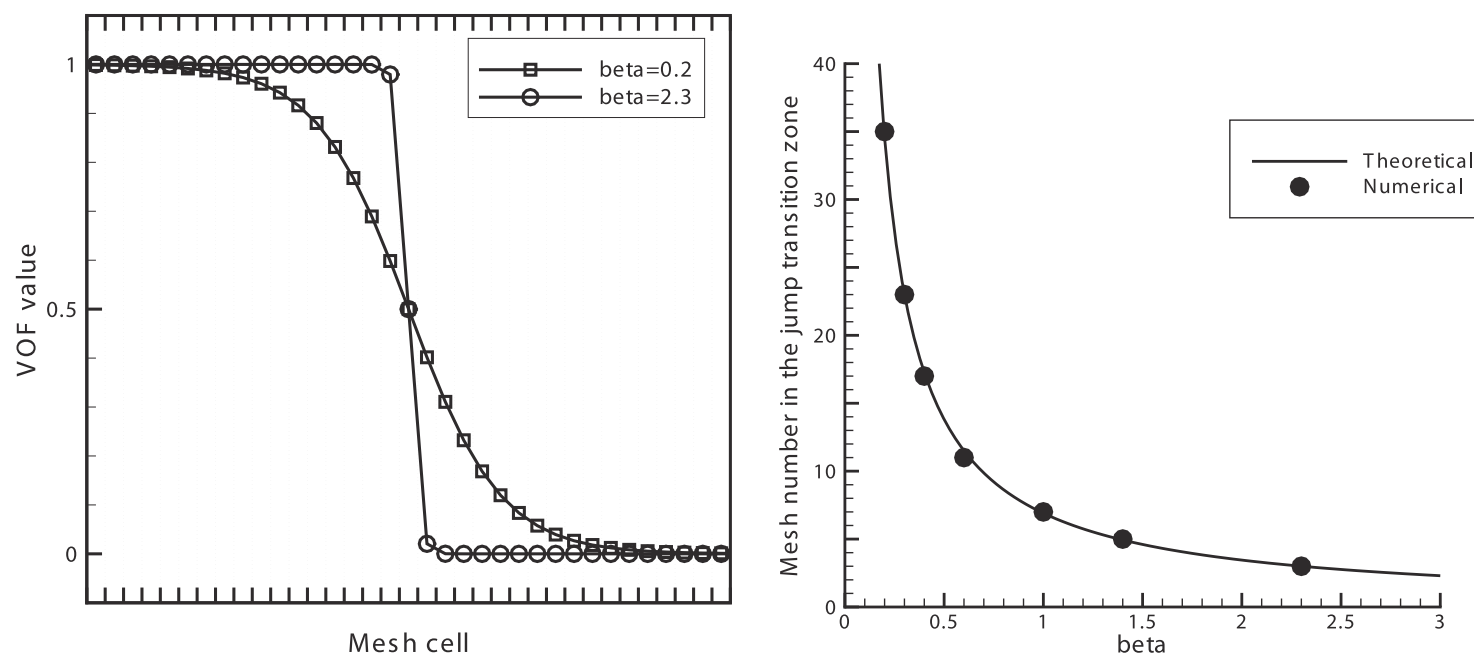

Fig. 1. Step jump computed by the THINC scheme (left) and the thickness of the jump transition zone as the function of $\beta$ (right).

mesh cells which satisfy $\epsilon \leqslant \bar{\phi}_{i} \leqslant 1-\epsilon$. The results are plotted in Fig. 1 (right) as the black circles. In fact, a theoretical relation between the slope parameter $\beta$ and the jump thickness can be obtained by solving the following equation

$$
\frac{1}{2}(1+\tanh (\beta \eta))=1-\epsilon,
$$

where $\eta=x / \Delta x$ is the normalized half thickness of the jump, and we assume a uniform grid spacing $\left(\Delta x=\Delta x_{i}\right)$ here. Given a value of $\beta$, we compute $\eta$ by

$$
\eta=\frac{1}{\beta} \tanh ^{-1}(1-2 \epsilon) \text {. }
$$

The solid line in Fig. 1 (right) shows the jump thickness $(2 \eta)$ computed by ( 8 ) as a function of $\beta$. It corresponds to the total number of mesh cells within the transition zone of the jump. It turns out that (8) gives an accurate estimation of the jump thickness computed by THINC scheme as the function of parameter $\beta$. For example, it is known from (8) that the jump can be resolved by approximately 2 to 3 mesh cells if $\beta=2.3$. It can be verified by the numerical result in Fig. 1(left).

\section{Improved multi-dimensional calculation}

The cause of ruffling the interface which aligns nearly in the direction of the velocity in the original THINC scheme is the use of the slope parameter $\beta$ as a constant in sweeps for all directions. Multi-dimensional flux approximations that use the SLIC (simple line interface calculation) reconstruction but consider the orientation of the interface have been reported in $[19,10]$. An interface orientation dependent weighting between the original THINC scheme and the first order donor cell scheme is suggested in [19], which significantly improves the geometrical accuracy of the original THINC scheme.

As we have seen from above, the jump thickness can be effectively controlled by $\beta$, a more reliable and elegant multidimensional scheme can be devised by determining $\beta$ adaptively according to the orientation of the interface.

We consider an interface having a unit normal, $n=\left(n_{x}, n_{y}\right)$, and a thickness in the normal direction, $2 \eta$, as shown in Fig. 2. To maintain the thickness in the normal direction, the hyperbolic tangent function constructed in the $x$ direction should have a jump thickness of $\eta_{x}=\eta /|\cos (\theta)|=\eta /\left|n_{x}\right|$ with $\theta$ being the direction angle to the $x$ axis, whereas that constructed in the $y$ direction should have a jump thickness of $\eta_{y}=\eta /|\sin (\theta)|=\eta /\left|n_{y}\right|$. From (8), the corresponding slope parameter should be

$$
\beta_{x}=\frac{1}{\eta_{x}} \tanh ^{-1}(1-2 \epsilon)=\frac{1}{\eta} \tanh ^{-1}(1-2 \epsilon)\left|n_{x}\right| \quad \text { and } \beta_{y}=\frac{1}{\eta_{y}} \tanh ^{-1}(1-2 \epsilon)=\frac{1}{\eta} \tanh ^{-1}(1-2 \epsilon)\left|n_{y}\right|
$$

respectively. If the jump is going to be resolved within 3 mesh cells, i.e. $\eta=1.5, \frac{1}{\eta} \tanh ^{-1}(1-2 \epsilon)$ values about 2.3. In practice, we estimate $\beta_{x}$ and $\beta_{y}$ by

$$
\beta_{x}=2.3\left|n_{x}\right|+0.01 \text { and } \beta_{y}=2.3\left|n_{y}\right|+0.01 .
$$

The unit normal $n=\left(n_{x}, n_{y}\right)$ is computed by differencing the VOF function $\bar{\phi}$. We use Parker and Youngs' algorithm described in [12]. The numerical flux for the $x$ direction is then calculated by replacing the slope parameter $\beta$ in (6) with $\beta_{x}$ given in (10), and the same applies to the $y$ direction. The scheme can be straightforwardly extended to 3 dimensions $(x, y, z)$ by adding another sweep in the $z$ direction with the corresponding slope parameter computed by $\beta_{z}=2.3\left|n_{z}\right|+0.01$. 


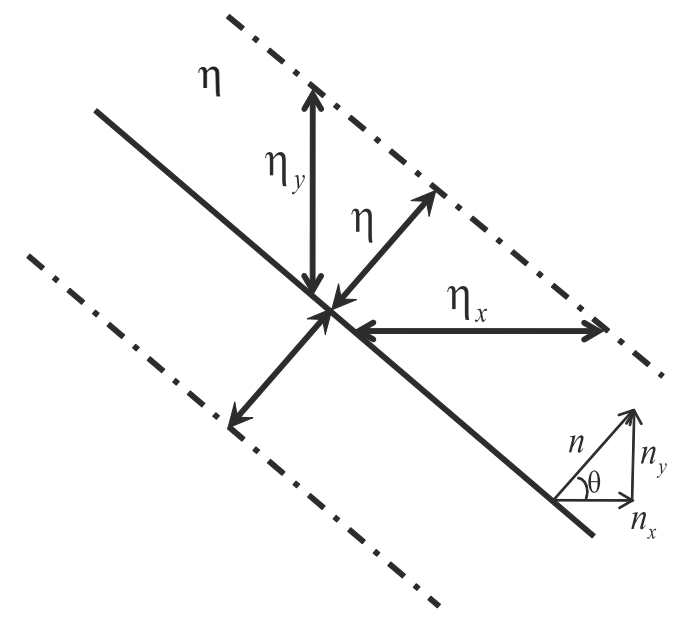

Fig. 2. The jump thickness in each dimension for multi-dimensional calculation.

\section{Numerical tests and discussions}

We verify the improved multi-dimensional THINC/SW scheme by some widely used benchmark tests for the VOF methods. Fig. 3 shows the results of a rotated slotted cylinder after one revolution known as the Zalesak's problem. It is found that the present scheme produces numerical solutions whose accuracy is comparable to those from the typical VOF schemes with PLIC reconstructions as given in [13] (Fig. 2) and [12] (Fig.11). Shown in the right panel of Fig. 3, the jump thickness, indicated by the contours between 0.05 and 0.95 , maintains compact within 2 to 3 mesh cells along the interface. A quantitative comparison is conducted by repeating the modified Zalesak slotted cylinder test suggested in [14] and used in other papers to evaluate different variants of VOF method with PLIC reconstructions.

The numerical error shown in Table 1 is defined by

$$
E_{R}=\frac{\sum_{i j}\left|\phi_{i j}-\tilde{\phi}_{i j}\right|}{\sum_{i j} \phi_{i j}}
$$

where $\phi_{i j}$ is the exact (initial) solution and $\tilde{\phi}_{i j}$ the numerical one. In order to compare with other existing VOF schemes, we also include the results for the FCT-VOF [14], Youngs' PLIC [14], DDR (Defined Donating Region) [6], ELVIRA/ $\Pi$ (efficient least squares volume-of-fluid interface reconstruction algorithm) [2] and GPCA (geometrical predictor-corrector advection) [4]. It shows that THINC/SW has an accuracy comparable to the typical PLIC schemes.

A deformed moving interface in the reversed single-vortex test described in [13] is computed. The velocity field is given by a time-dependent stream function on a unit square,

$$
\Psi(x, y, t)=\frac{1}{\pi} \sin ^{2}(\pi x) \sin ^{2}(\pi y) \cos \left(\frac{\pi t}{T}\right) .
$$
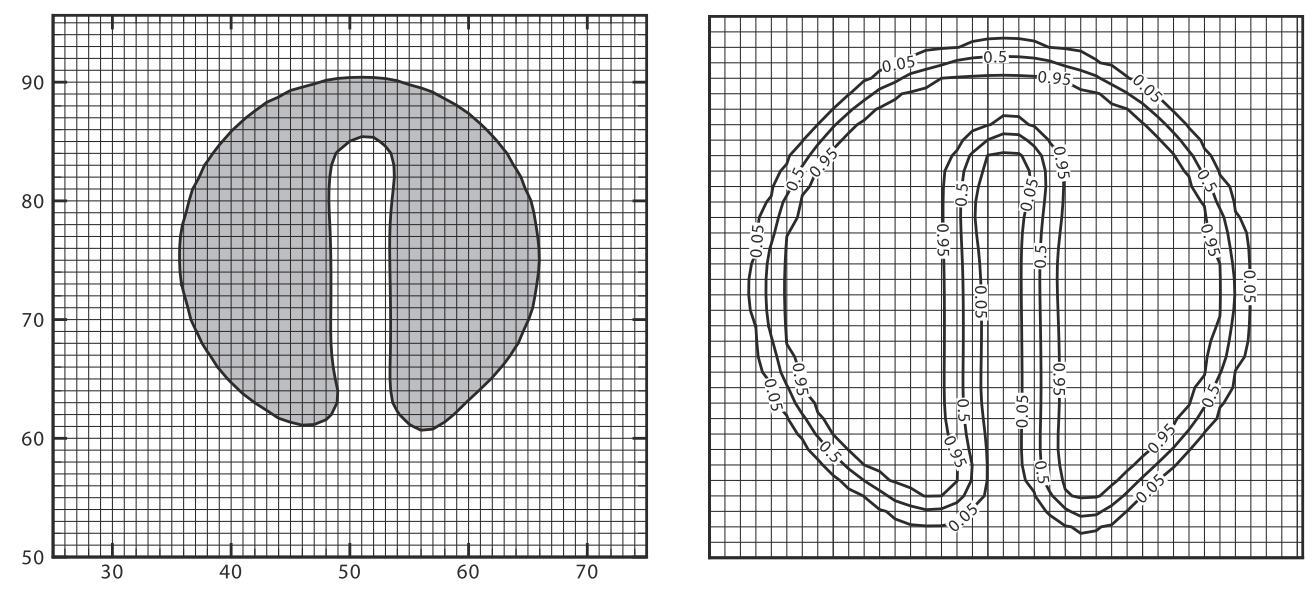

Fig. 3. Numerical results of Zalesak's slotted-cylinder test problem on a $100 \times 100$ grid after one revolution. The interface ( 0.5 contour) is shown in the left panel, and an enlarged view of $0.05,0.5$ and 0.95 contours is plotted in the right panel. 
Table 1

Numerical errors of the modified Zalesak slotted cylinder test.

\begin{tabular}{lr}
\hline Algorithms & Error $\left(E_{R}\right)$ \\
\hline FCT-VOF [14] & $3.29 \times 10^{-2}$ \\
DDR/Youngs [6] & $1.56 \times 10^{-2}$ \\
DDR/Puckett [6] & $1.50 \times 10^{-2}$ \\
Youngs [14] & $1.09 \times 10^{-2}$ \\
ELVIR/П [2] & $1.00 \times 10^{-2}$ \\
GPCA [4] & $9.79 \times 10^{-3}$ \\
THINC [16] & $3.52 \times 10^{-2}$ \\
THINC/WLIC [19] & $1.96 \times 10^{-2}$ \\
THINC/SW & $1.34 \times 10^{-2}$ \\
\hline
\end{tabular}

A circle of radius 0.15 is initially centered at $(0.5,0,75)$. The circle is transported by THINC/SW scheme up to $T=8$. The numerical results at $T / 2$ and $T$ are presented in Fig. 4 . The interface has been deformed into a spiral at $T / 2$ and is well resolved except that the thin tail is broken into small droplets in a way quite similar to all other VOF schemes. We enlarge one droplet in Fig. 4(left) to show the interface thickness by contours of 0.05, 0.5 and 0.95 . It reveals that even heavily distorted interface can maintain a compact thickness of 2 to 3 mesh cells. The same is observed from the contoured interface depicted in Fig. 4(right) at $T$ as well. The $L_{1}$ error defined by

$$
E_{1}=\sum_{i j}\left|\phi_{i j}-\tilde{\phi}_{i j}\right| \Delta x \Delta y \text {, }
$$

is shown in Table 2 in comparison with THINC/WLIC scheme. The two schemes have equivalent numerical accuracy. The numerical errors of both schemes are somewhat larger than those of the PLIC schemes as shown in [13,4] but still of the same order. It is observed that the two THINC schemes converge at nearly second order rate under adequate grid resolutions. It should be notified that the THINC schemes exactly conserves the VOF function $\phi$. Determining the interface from the computed $\phi$ field, however, remains some arbitrariness. In practice, the interface can be reasonably identified by the 0.5 contour surface. We examined the volume of the mesh cells encompassed by the 0.5 -contour surface of the numerical solution in the reversed single-vortex test, and found that the total volume experiences a change less than $1.2 \%$ through the whole computation. Because the interface thickness remains constantly within 2 to 3 mesh cells, the volume conservation can be more accurately retrieved by identifying the interface as the contour of a variable value in computations.

We also tested the 3D scheme with the vortical deformal flow field given in [8]. A sphere with a radius of 0.15 centered at $(0.35,0.35,0.35)$ is deformed by a three dimensional reversing velocity field in a unit cube,

$$
\left\{\begin{array}{l}
u(x, y, z)=2 \sin ^{2}(\pi x) \sin (2 \pi y) \sin (2 \pi z) \cos (\pi t / T) \\
v(x, y, z)=-\sin (2 \pi x) \sin ^{2}(\pi y) \sin (2 \pi z) \cos (\pi t / T) \\
w(x, y, z)=-\sin (2 \pi x) \sin (\pi y) \sin ^{2}(\pi z) \cos (\pi t / T)
\end{array}\right.
$$

The period is set to $T=3$ in the present test. The results for two grid resolutions, $100^{3}$ and $200^{3}$, are shown in Fig. 5 . The geometrical faithfulness of the numerical solutions are comparable to other PLIC schemes (see for example Fig. 9 in [9]).
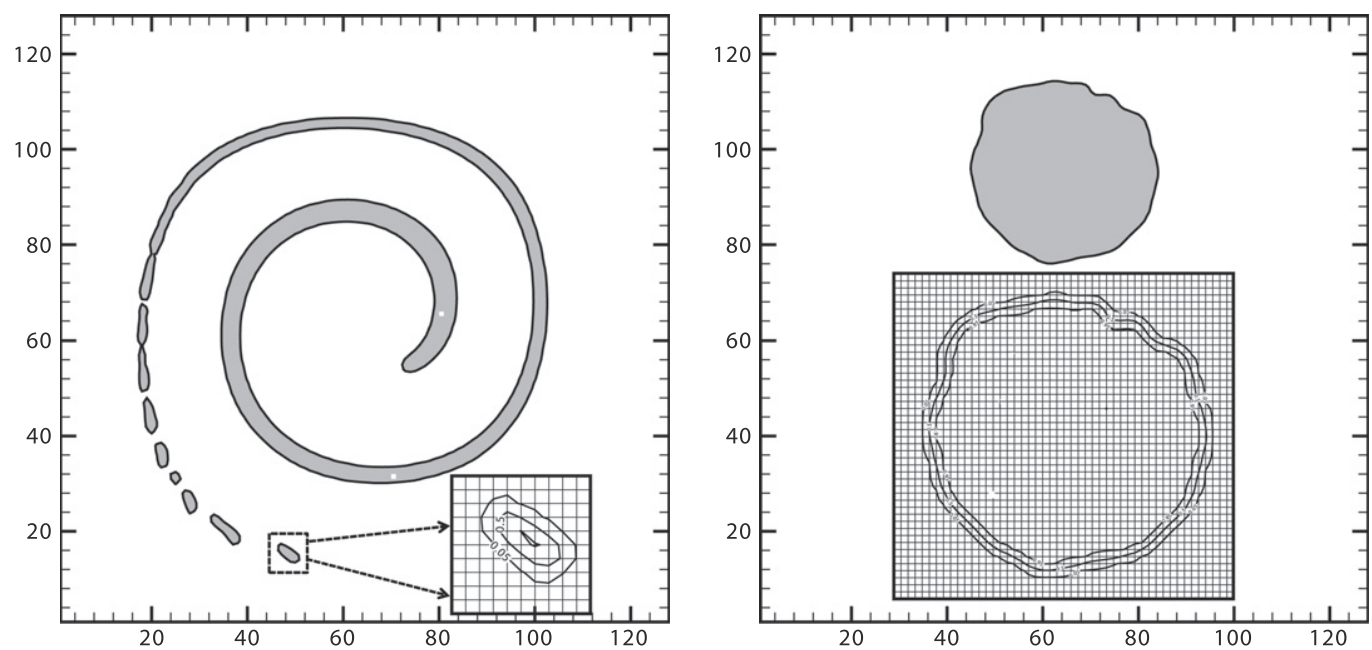

Fig. 4. The interface of the Rider-Kothe reversed single-vortex test at $T / 2$ (left) and $T$ (right) with $T=8$ on a $128 \times 128$ grid. 
Table 2

Numerical errors $\left(E_{1}\right)$ and convergence rates for the reversed single-vortex test [13] with $T=8$.

\begin{tabular}{llllll}
\hline Grid resolutions & $32^{2}$ & Rate & $64^{2}$ & Rate & $128^{2}$ \\
\hline THINC/WLIC & $4.16 \times 10^{-2}$ & 1.37 & $1.61 \times 10^{-2}$ & 2.18 & $3.56 \times 10^{-3}$ \\
THINC/SW & $3.90 \times 10^{-2}$ & 1.36 & $1.52 \times 10^{-2}$ & 1.94 & $3.96 \times 10^{-3}$ \\
\hline
\end{tabular}

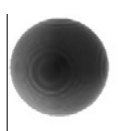

Initial(Exact)

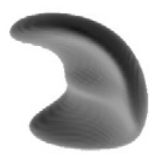

$\mathrm{T} / 6$

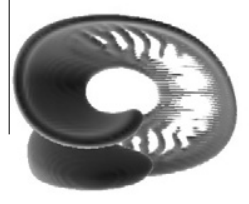

$\mathrm{T} / 2$

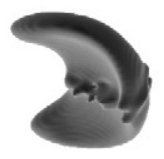

$5 \mathrm{~T} / 6$

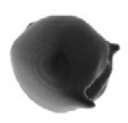

$\mathrm{T}=3$

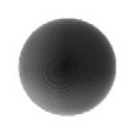

Initial(Exact)

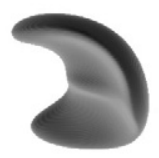

$\mathrm{T} / 6$

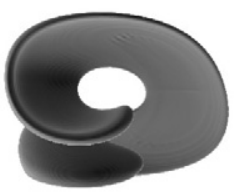

$\mathrm{T} / 2$

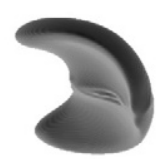

$5 \mathrm{~T} / 6$

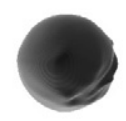

$\mathrm{T}=3$

Fig. 5. A sphere deformed by a reversed 3D vortical flow on a $100^{3}$ grid (left column) and a $200^{3}$ grid (right column). Displayed are the snapshots at $0, T / 6, T /$ $2,5 T / 6$ and $T=3$.

The $L_{1}$ errors of numerical solutions and the corresponding convergence rates are given in Table 3 . We include the numerical results of the RK-3D (the 3D version of Rider and Kothe PLIC scheme [13]) and the FMFPA-3D (face-matched flux polyhedron advection) given in [7] for comparison. Overall, the numerical errors are slightly larger than these PLIC VOF schemes, but still comparable.

In summary, an improved multi-dimensional THINC scheme is devised based on the observation that the thickness of the jump transition layer can be well maintained and predetermined by the steepness parameter in the 1D THINC buiding block. Numerical tests reveal that it has a numerical accuracy comparable to some of the existing VOF schemes that use geometrical interface reconstructions. The compact thickness constantly kept for even heavily deformed interface makes the present scheme significantly superior to other algebraic type VOF schemes [14,17]. Without geometrical reconstruction, the scheme is very simple and easy to use. We expect it to be a practical interface capturing scheme of great simplicity and adequate numerical accuracy. 
Table 3

Numerical errors and convergence rates for 3D reversed single-vortex test [8] with $T=3$.

\begin{tabular}{lllll}
\hline Grid resolutions & $32^{3}$ & Rate & $64^{3}$ & $128^{3}$ \\
\hline RK-3D[7] & $7.85 \times 10^{-3}$ & 1.51 & $2.75 \times 10^{-3}$ & 1.89 \\
FMFPA-3D[7] & $7.44 \times 10^{-3}$ & 1.42 & $2.79 \times 10^{-3}$ & 1.97 \\
THINC/SW & $8.39 \times 10^{-3}$ & 1.27 & $3.47 \times 10^{-3}$ & $7.14 \times 10^{-4}$ \\
\hline
\end{tabular}

\section{References}

[1] A. Ashgriz, J.P. Poo, FLAIR: flux line-segment model for advection and interface reconstruction, J. Comput. Phys. 93 (1991) $449-468$.

[2] E. Aulisa, S. Manservisi, R. Scardovelli, S. Zaleski, A geometrical area-preserving volume-of-fluid advection method, J. Comput. Phys. 192 (2003) 355364.

[3] E. Aulisa, S. Manservisi, R. Scardovelli, S. Zaleski, Interface reconstruction with least-squares fit and split advection in three-dimensional Cartesian geometry, J. Comput. Phys. 225 (2007) 2301-2319.

[4] A. Cervone, S. Manservisi, R. Scardovelli, S. Zaleski, A geometrical predictor-corrector advection scheme and its application to the volume fraction function, J. Comput. Phys. 228 (2009) 406-419.

[5] D.J.E. Harvie, D. F Fletcher, A new volume of fluid advection algorithm: the stream scheme, J. Comput. Phys. 162 (2000) 1-32.

[6] D.J.E. Harvie, D. F Fletcher, A new volume of fluid advection algorithm: the defined donating region scheme, Int. J. Numer. Methods Fluids 35 (2001) $151-172$.

[7] J. Hernandez1, J. Lopez, P. Gomez, C. Zanzi, F. Faura, A new volume of fluid method in three dimensions - Part I: multidimensional advection method with face-matched flux polyhedra, Int. J. Numer. Methods Fluids 58 (2008) 897-921.

[8] R. LeVeque, High-resolution conservative algorithms for advection in incompressible flow, SIAM J. Numer. Anal. 33 (1996) 627-665.

[9] J. Lopez, P. Gomez, C. Zanzi, F. Faura, J. Hernandez, A new volume of fluid method in three dimensions - Part II: piecewise-planar interface reconstruction with cubic-Bezier fit, Int. J. Numer. Methods Fluids 58 (2008) 923-944.

[10] M. Marek, W. Aniszewski, A. Boguslawski, Simplified volume of fluid method (SVOF) for two-phase flows, TASK Quarter 12 (2008) $255-265$.

[11] D. Pan, C.H. Chang, The capturing of free surfaces in incompressible multi-fluid flows, Int. J. Numer. Methods Fluids 30 (2000) $203-222$.

[12] J.E. Pilliod Jr., E.G. Puckett, Second-order accurate volume-of-fluid algorithms for tracking material interfaces, J. Comput. Phys. 199 (2004) 465-502.

[13] W.J. Rider, D.B. Kothe, Reconstructing volume tracking, J. Comput. Phys. 141 (1998) 112-152.

[14] M. Rudman, Volume-tracking methods for interfacial flows calculations, Int. J. Numer. Methods Fluids 24 (1997) 671-691.

[15] C.D. Sijoy, S. Chaturvedi, Volume-of-fluid algorithm with different modified dynamic material ordering methods and their comparisons, J. Comput. Phys. 229 (2010) 3848-3863.

[16] F. Xiao, Y. Honma, T. kono, A simple algebraic interface capturing scheme using hyperbolic tangent function, Int. J. Numer. Methods Fluid 48 (2005) $1023-1040$.

[17] S.N. Yakovenko, K.C. Chang, Volume fraction flux approximation in a two-fluid flow, Thermophys. Aeromech. 15 (2008) $2169-2186$.

[18] D.L. Youngs, Time-dependent multi-material flow with large fluid distortion, in: K.W. Morton, M.J. Baines (Eds.), Numerical Methods for Fluid Dynamics, vol. 24, Academic, New York, 1982, pp. 273-285.

[19] K. Yokoi, Efficient implementation of THINC scheme: a simple and practical smoothed VOF algorithm, J. Comput. Phys. 226 (2007) 1985-2002. 